\title{
The present and potential future methods for delivering CRISPR/Cas9 components in plants
}

Dulam Sandhya ${ }^{1 \dagger}$, Phanikanth Jogam ${ }^{1 \dagger}$, Venkateswar Rao Allini ${ }^{1}$, Sadanandam Abbagani ${ }^{{ }^{*}}$ and Anshu Alok ${ }^{2^{*}}$

\begin{abstract}
Background: CRISPR/Cas9 genome editing technology is a DNA manipulation tool for trait improvement. This technology has been demonstrated and successfully applied to edit the genome in various species of plants. The delivery of CRISPR/Cas9 components within rigid plant cells is very crucial for high editing efficiency. Here, we insight the strengths and weaknesses of each method of delivery.

Main text: The mutation efficiency of genome editing may vary and affected by different factors. Out of various factors, the delivery of CRISPR/Cas9 components into cells and genome is vital. The way of delivery defines whether the edited plant is transgenic or transgene-free. In many countries, the transgenic approach of improvement is a significant limitation in the regulatory approval of genetically modified crops. Gene editing provides an opportunity for generating transgene-free edited genome of the plant. Nevertheless, the mode of delivery of the CRISPR/Cas9 component is of crucial importance for genome modification in plants. Different delivery methods such as Agrobacterium-mediated, bombardment or biolistic method, floral-dip, and PEG-mediated protoplast are frequently applied to crops for efficient genome editing.
\end{abstract}

Conclusion: We have reviewed different delivery methods with prons and cons for genome editing in plants. A novel nanoparticle and pollen magnetofection-mediated delivery systems which would be very useful in the near future. Further, the factors affecting editing efficiency, such as the promoter, transformation method, and selection pressure, are discussed in the present review.

Keywords: CRISPR/Cas9, Floral-dip, PEG, Pollen magnetofection, Genome editing

\section{Background}

Plants play a vital role in human life by offering a variety of plant-based products such as fruits, food grains, vegetables, and medicine. The various traits of plants can be improved by plant breeding and genetic engineering activities [1]. Plant genetic engineering can be achieved utilizing multiple tools such as overexpression, RNA interference, Zinc finger TALEN nuclease, and CRISPR/ Cas9 [2-5]. CRISPR/Cas9 genome editing tool is derived

\footnotetext{
* Correspondence: nandamas@gmail.com; anshualok2@gmail.com

${ }^{\dagger}$ Dulam Sandhya and Phanikanth Jogam contributed equally to this work. 'Department of Biotechnology, Kakatiya University, Warangal, Telangana, India

${ }^{2}$ Department of Biotechnology, UIET, Panjab University, Chandigarh, India
}

from the bacterial CRISPR system, which is known to involve in the immune system. CRISPR/Cas9 technology has gained tremendous popularity due to its specificity and efficiency in editing the genome. Several CRISPR/ Cas9 and its variants have been applied for the genome editing of many desired genes. The CRISPR (clustered regularly interspaced short palindromic repeats) locus and its associated proteins are basically found in few bacteria, and it is related to immunity against phages. The different regions of CRISPR locus transcribed and lead to the formation of CRISPR RNA (crRNA) and trans-activating CRISPR RNA (tracrRNA). The crRNA, tracrRNA, and Cas9 encounter the phage DNA. The

\section{Springer Open}

(ㅇ The Author(s). 2020 Open Access This article is licensed under a Creative Commons Attribution 4.0 International License, which permits use, sharing, adaptation, distribution and reproduction in any medium or format, as long as you give appropriate credit to the original author(s) and the source, provide a link to the Creative Commons licence, and indicate if changes were made. The images or other third party material in this article are included in the article's Creative Commons licence, unless indicated otherwise in a credit line to the material. If material is not included in the article's Creative Commons licence and your intended use is not permitted by statutory regulation or exceeds the permitted use, you will need to obtain permission directly from the copyright holder. To view a copy of this licence, visit http://creativecommons.org/licenses/by/4.0/. 
guide RNA (gRNA) is a synthetic gene comprised of crRNA and tracrRNA [6].

The Cas9 gene and gRNA under the regulation of the appropriated promoters within any vector, can be delivered into the plant cells. In another approach, the Cas9, also known as RNA guided site-specific nucleases (RGNs) and transcribed gRNA, is assembled and then delivered into plant regenerative tissue. The target site must contain 5'NGG3' for the action of the CRISPR/Cas9 system, which is also known as Protospacer Adjacent Motif (PAM). Cas9 cleaves both the strands of a target gene or DNA with the help of gRNA. This double-stranded breaks (DSBs) may be restored by either homologous direct repair (HDR) or non-homologous end joining (NHEJ) via a repair mechanism [7]. During DNA repair, the insertion or deletion of nucleotide results in the point mutation or frameshift mutation. These mutations are generally identified by various techniques; however, the restriction enzyme site loss assay, AFLP, and Sanger-based sequencing are frequently used $[8,9]$.

Different strategies have been developed to target multiple genes at a time, i.e., multiplexing [10, 11]. This multiplex genome engineering is generally used to target various genes within the genome or distinct target within one gene to increase mutation efficiency. This technique involves the expression of multiple gRNAs under different promoters or single promoter using the polycistronic gRNA unit [11]. Polycistronic gRNA unit is assembled with the help of either tRNA-gRNA or Cys4gRNA. This single synthetic gene is transcribed by a single promoter. The RNases $\mathrm{P}$ and $\mathrm{Z}$ enzymes cleave polycistronic tRNA-gRNA. Csy4 (CRISPR system yersinia 4) is an RNA nuclease characterized from Pseudomonas aeruginosa separate polycistronic Cys4-gRNA into individual gRNA [11]. The tRNA processing enzymes are naturally present in almost all living organisms, including plant cells [12]. This technology has been demonstrated and applied in various plants such as Arabidopsis, tobacco, potato, tomato, rice, wheat, and banana [4, 5, 13, 14]. Targeting various genes by employing CRISPR/Cas9 is a more relaxed approach in comparison to the other known genome modification tools. Therefore, it is considered as a most promising tool for metabolic engineering.

The delivery of CRISPR/Cas9 components within rigid plant cells is a tough task. There are three methods of the construct delivery in plant cell: PEG mediated, Agrobacterium-mediated transformation, and bombardment or biolistic transformation. However, we insights the strength and weaknesses of each method of delivery depend upon plant species. We have elaborately discussed two potential methods for CRISPR/Cas9 vector-nanoparticle complex and a novel pollen magnetofection-mediated delivery in plants that would be most useful shortly (Fig. 1).

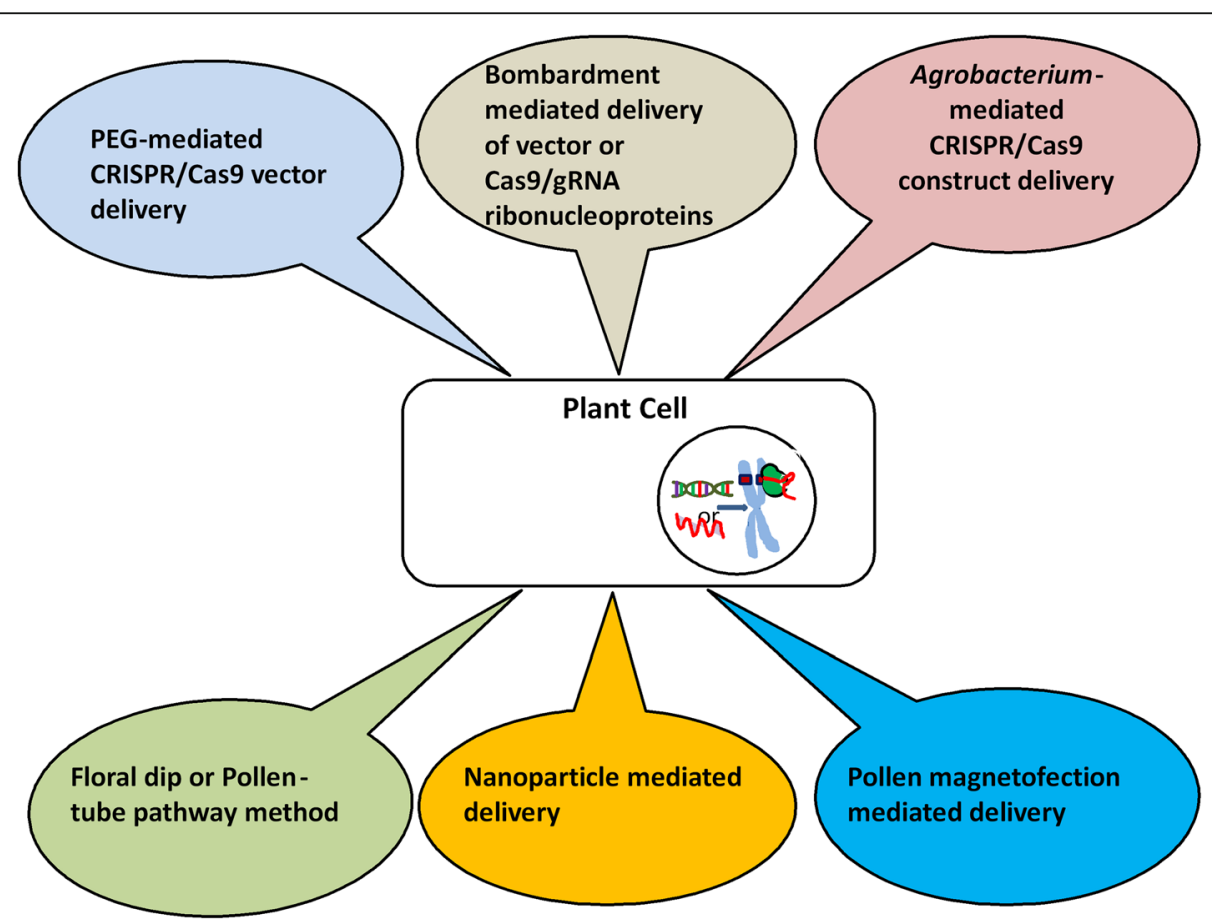

Fig. 1 Existing and potential future CRISPR/Cas9 delivery methods. Different well-known delivery methods such as Agrobacterium-mediated delivery, Bombardment-mediated delivery, PEG-mediated delivery, and floral dip or pollen-tube tube pathway method. Potential pollen magnetofection-mediated delivery and nanoparticle-mediated delivery will be useful in near future to avoid tissue culture 


\section{Main text}

\section{PEG-mediated CRISPR/Cas9 vector delivery}

This is an important genetic transformation method carried out in the presence of polyethylene glycol (PEG) (Fig. 2). This method is successfully performed in protoplasts of different plants such as in maize, soybean, A. thaliana, tobacco, rice, and wheat, as summarized in Table 1. The plasmid-containing Cas 9 and gRNA is incubated with protoplast in the presence of PEG. The PEG-mediated CRISPR construct delivery was firstly reported in maize and in this U3, and CaMV35S promoters were used for gRNA and Cas9, respectively [25]. In a few studies, Cas9 was expressed under some specific promoters, designed for specific plants, and to target crucial gene [27]. Plasmid DNA is dissolved in water and filtered to make it sterile and then mixed with protoplast suspension. After a few minutes, the required concentration of PEG is mixed slowly to protoplasts. After that, protoplasts are regenerated with a suitable regeneration medium [28]. Cas9/ gRNA ribonucleoproteins were used to make transgenefree mutant plants in rice, Arabidopsis, tobacco, and lettuce using PEG-mediated delivery. The editing frequency in lettuce mutants was up to $46 \%$ [29]. Later, Kim et al. used Cpf1/CrRNA ribonucleoproteins to edit the genome of soybean and tobacco without using vector or DNA [30]. Cpf1 (CRISPR from Prevotella and Francisella 1) is an endonuclease of class II and type V CRISPR system. It identifies a thymidine rich PAM (TTTN) in a target location. Cpf1-mediated genome editing requires only crRNA, whereas Cas9 requires both crRNA and tracerRNA [30].

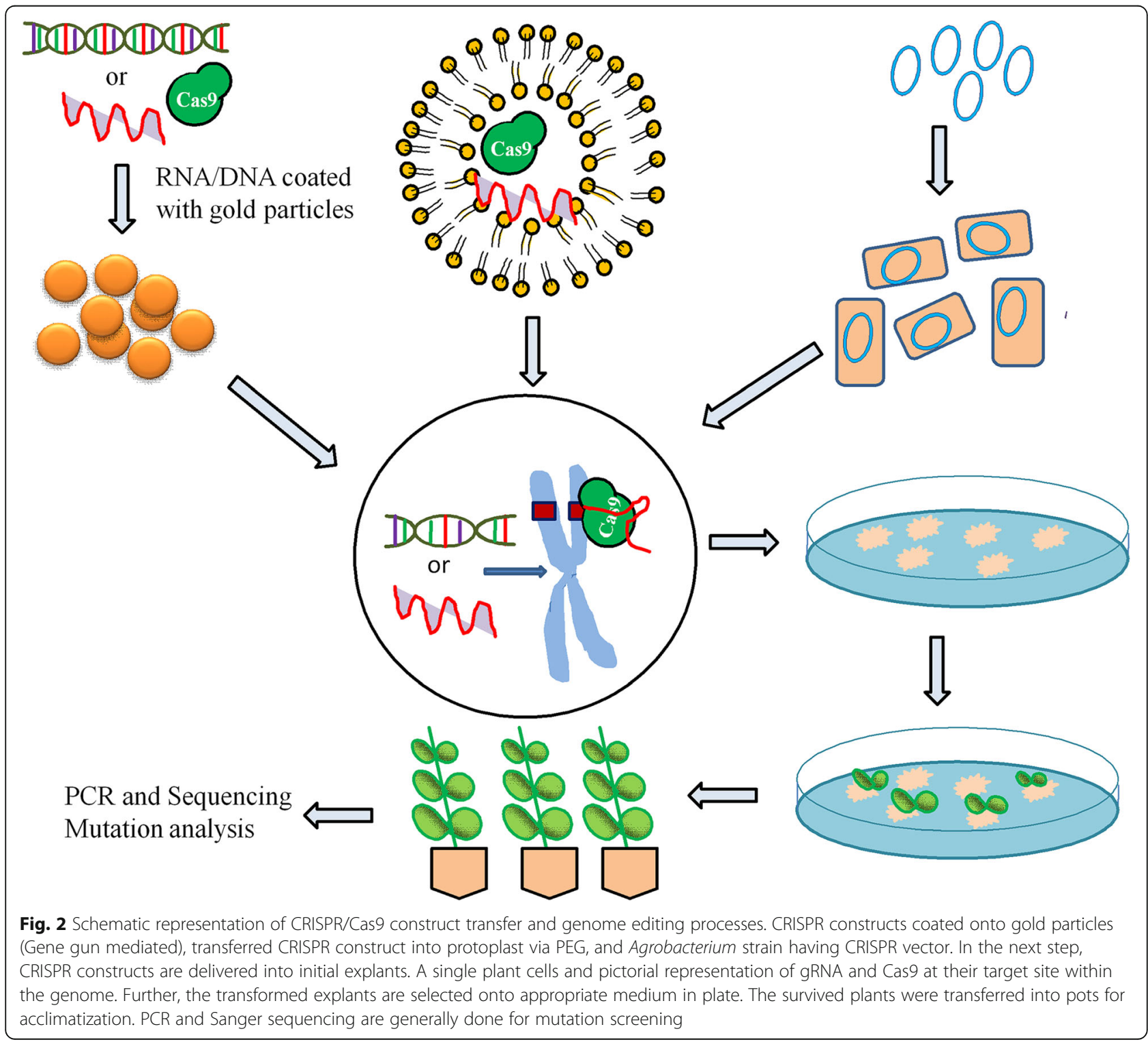


Table 1 PEG mediated CRISPR/Cas9 components delivery into different plants

\begin{tabular}{|c|c|c|c|}
\hline Plant name & CRISPR/Ca9 vector or ribonucleoprotein complexes & Targeted genes & Reference \\
\hline Apple & Cas9-sgRNA ribonucleoprotein complexes & DIPM-1, 2, 4 & [15] \\
\hline Brassica oleracea, Brassica rapa & Cas9-sgRNA ribonucleoprotein complexes & FRI, PDS & [16] \\
\hline Citrullus lanatus & PHSN1, PHSN2 & CIPDS & {$[17]$} \\
\hline Glycine max & pCas9-GmU6-sgRNA, pCas9-AtU6-sgRNA & Glyma08g02290, Glyma12g37050, Glyma06g14180 & [18] \\
\hline Grapevine & Cas9-sgRNA ribonucleoprotein complexes & MLO-7 & {$[15]$} \\
\hline Oryza sativum & pRGE3, pRGE6 & OSMPK5 & [19] \\
\hline Oryza sativum & puC19-OsCas9 & OSSWEET14, OsSWEET11 & {$[20]$} \\
\hline Oryza sativum & pJIT163-2NLSCas9 & OsPDS, OsBADH2 & [21] \\
\hline Petunia & Cas9-sgRNA ribonuclease protein complexes (RNPs) & PhACO1 & [22] \\
\hline Physcomitrella patens & pAct-Cas9, psgRNA PpAPT-KO4, PpAPT-KO7 & PPAPT & [23] \\
\hline Solanum tuberosum & Cas9-sgRNA Ribonucleoprotein complexes (RNPs) & GBSS (GT4) & [4] \\
\hline Triticum aestivum & pCR8-U6-gRNA & TaEPSPS & [24] \\
\hline Zea mays & p ZmU3-gRNA & $Z m I P K$ & {$[25]$} \\
\hline Zea mays & CT-nCas9 & $Z m A L S 1, Z m A L S 2$ & {$[26]$} \\
\hline
\end{tabular}

The main strength of the PEG-mediated delivery method is widely used to deliver Cas9/gRNA ribonucleoproteins. The vector less or DNA-free edited mutants of plants will be more acceptable and probably no issue with regulatory and ethical barriers. In 2016, transgenefree editing of six polyphenol oxidase mushroom was generated using ribonucleoproteins and PEG mediated. The mutant mushroom showed a $30 \%$ reduced enzyme activity responsible for browning, and it also escapes US regulation [31]. This ribonucleoprotein complexes cannot be delivered by widely used Agrobacterium mediated or floral-dip method.

The significant challenges and weakness of PEGmediated delivery method is the establishment of suspension cells and protoplasts isolation. Apart from this, the main hurdle of regeneration of protoplasts into whole plants is in the case of recalcitrant plants. Due to this reason, the alternate methods of delivery of Cas9/gRNA ribonucleoproteins require to explore for efficient genome editing.

\section{Bombardment-mediated delivery of vector or Cas9/gRNA ribonucleoproteins}

This method of transformation or gene transfer needs a device known as "gene gun or biolistic gun". Generally, gold, silver, and tungsten particles are used as the carrier for vectors or Cas9/gRNA ribonucleoproteins (Fig. 2). CRISPR/Cas9 components are transferred through coated particles into explants by applying high pressure. This method requires optimized conditions such as helium pressure, target distance, particle size, and type of explants used. The transformed explants are regenerated onto regeneration medium with appropriate selection pressure. The successful delivery of Cas9/gRNA ribonucleoproteins and successive regeneration of mutants have been reported in maize [32], potato [4], and brassica [16], as tabulated in Table 2. Due to vector/DNA less editing, the Cas9/gRNA ribonucleoprotein delivery by the bombardment method is in demand. The regeneration of transformed tissues and selection pressure is tedious, and therefore, very low editing efficiency is

Table 2 Particle bombardment method for CRISPR/Cas9 component delivery

\begin{tabular}{lllll}
\hline Plant name & CRISPR/Cas9 vector or RNP complex & Selectable marker & Target genes & Reference \\
\hline Glycine max & QC810 and RTW830, QC799 and RTW831 & Hptll & DD20, DD43 & [33] \\
Hordeum vulgare & pcas9:SgRNA & Hptll & ENGase & [34] \\
Oryza sativum & pCam1300-CRISPR-B & Hptll & crtl, ZmPsy & [35] \\
Oryza sativum & CRISPR-RNP complex & Hptll & OsPDS1 & {$[36]$} \\
Oryza sativum & pJIT163-2NLSCas9 & Hptll & OsPDS, OsBADH2 & {$[21]$} \\
Oryza sativum & pOsU3-SgRNA, pJIT163-2NLSCas9 & Hptll & OsPDS, OsDEP1 & [37] \\
Triticum aesituvam & pJT163-Ubi & bar & TaMLO-A1, TaMLO-B1, TaMLO-D1 & [38] \\
Zea mays & pSB11-Ubi:Cas9 & Pat & LIG1, Ms26, Ms45, ALS1, ALS2 & [32] \\
\hline
\end{tabular}


achieved in maize 2.4 to $9.7 \%$ [32]. Recently, Cas9 with cytidine base editor along gRNA was in vitro transcribed and used for delivery into protoplast of wheat and rice for base editing [39].

The main strength of this delivery method is there is no necessity of the CRISPR/Cas9 binary vector. Several types of explants can be transformed with large and multiple DNA and RNA can be delivered. The most important is the Cas9/gRNA ribonucleoprotein complex which can also be delivered efficiently. The main drawback of this delivery method is random integration patterns within the genome, relatively less editing efficiency, costlier than others, and the bombardment sites such as cytoplasm, nucleus, mitochondria, or plastid cannot be controlled [40].

\section{Agrobacterium-mediated CRISPR/Cas9 construct delivery} Out of all delivery methods, this is the most widely used for a wide range of plant species. The binary vector that contains Cas9 and the gRNA expression cassette is transformed into the Agrobacterium strain. Further, the Agrobacterium-mediated genetic transformation of CRISPR constructs into the desired explant such as callus, leaf, and floral organs of plants (Fig. 2). To date, more than 20 plant species were efficiently edited with the help of Agrobacterium-mediated delivery of CRISPR/ Cas9 components, summarized in Table 3. Agrobacterium-mediated transformation is more efficient and showed high editing efficiency than the particle bombardment method. Monocot crops that have less regeneration and transformation capacity are also frequently used for genetic transformation utilizing Agrobacterium. The CRISPR/Cas9 binary vectors suitable for Agrobacterium for high editing efficiency for monocot and dicot were designed [61]. Agrobacterium-mediated genome editing of banana cultivar Rasthali showed 59\% mutation frequency of the phytoene desaturase gene [13]. In

Table 3 Agrobacterium-mediated delivery of CRISPR/Cas9 components in different plant species

\begin{tabular}{|c|c|c|c|c|c|}
\hline Plant name & CRISPR/Cas9 vector & $\begin{array}{l}\text { Selectable } \\
\text { marker }\end{array}$ & Strain & Target genes & Reference \\
\hline Arabidopsis thaliana & pUC119-RCS & Marker free & GV3101 & AtPDS3, AtFLS2, RACK1b, RACK1C & [41] \\
\hline Arabidopsis thaliana & pCAMBIA1300 & Hptll & GV3101 & $B R I 1, G A l, J A Z 1$ & [42] \\
\hline Banana & pRGEB31 & Hptll & AGL1 & RAS-PDS & [13] \\
\hline Banana & pRGEB31 & Hptll & AGL1 & $L C Y \varepsilon$ & [43] \\
\hline Citrus sinensis & pCas9-GN & Nptll & LBA4404 & CsWRKY22 & [44] \\
\hline Cucumis sativum & pRCS & Nptll & EHA105 & elF4E, elF(iso) $4 E$ & {$[45]$} \\
\hline Glycine max & p201N Cas9 & Nptll & K599 & GFP transgene & [46] \\
\hline Kiwi fruit & $\begin{array}{l}\text { pHLW-sgRNA-Cas9-AtU6-1, pPTG-sgRNA-Cas9- } \\
\text { U6-1 }\end{array}$ & Nptll & EHA105 & ACPDS & [47] \\
\hline Lotus japonicus & pCAMBIA1300 & Hptll & EHA105 & LjLb1, LjLb2, LjLb3, LjSYMRK & [48] \\
\hline $\begin{array}{l}\text { Marchantia } \\
\text { polymorpha }\end{array}$ & pMpGE013 and pMpGE014 & Hptll & - & MpARF1 & [49] \\
\hline Medicago trancatula & pMDC32-AtU6-26 & Hptll & ARqual & GUS & {$[50]$} \\
\hline Medicago truncatula & pFGC5941 & Bar & - & MtPDS & [51] \\
\hline $\begin{array}{l}\text { Nicotiana } \\
\text { benthamaina }\end{array}$ & $\mathrm{plCH} 86966$ & - & AGL1 & NbPDS, PDS & [14] \\
\hline $\begin{array}{l}\text { Nicotiana } \\
\text { benthamaina }\end{array}$ & pUC19, pKQ334 & Hptll & GV3101 & $\mathrm{NbPDS3}, \mathrm{NblspH}$ & {$[52]$} \\
\hline Nicotiana tabaccum & PORE & Nptll & LBA4404 & NtPDS, NtPDR6 & [53] \\
\hline Oryza sativum & VK005 & Hptll & EHA105 & $\mid S A 1$ & [54] \\
\hline Populous tomentosa & pYLCRIPSR/Cas9, pUC18 & Hptll & - & PtoPDS & {$[55]$} \\
\hline Salvia miltiorrhiza & pCAMBIA1300 & Hptll & C58C1 & SmRAS & {$[56]$} \\
\hline Solanum lycopersicum & pYLCRISPR/Cas9 & Hptll & - & $S G R 1, L C Y-E, B I C, L C Y-B 1, L C Y-B 2$ & {$[57]$} \\
\hline Solanum lycopersicum & pENTR-sgRNA: pMR290/Cas9 & Nptll & EHA105 & SICCD8 & {$[58]$} \\
\hline Solanum tuberosum & pMDC32 & Hptll & - & StALS1 & {$[59]$} \\
\hline Sorghum bicolor & pVS1 binary vector derived from pLH7500 & Nptll & Y158 & DsRED2 & {$[20]$} \\
\hline Triticum aesituvam & $\mathrm{pB} \mid 121$ & Nptll & GV3101 & Inox, PDS & {$[14]$} \\
\hline Zea mays & pMCG1005 & Bar & EHA101 & $\begin{array}{l}\text { Argonaute 18, Dihydroflavonol-4- } \\
\text { reductase }\end{array}$ & {$[60]$} \\
\hline
\end{tabular}


another report, $100 \%$ editing efficiency was reported in Cavendish banana cultivar "Williams" using Agrobacterium-mediated editing of the same gene [62]. Out of all delivery methods, Agrobacterium mediated is the most promising and useful method even for woody plants. The woody plants such as Citrus sinensis and poplar were efficiently edited by Agrobacterium-mediated genome editing $[63,64]$. These methods are generally useful for the plant that is easily transformed through the leaf, callus, and floral organs.

The significant advantage of this method is high editing efficiency compared to other known methods. Another advantage of this method is broad applicability, easily available, and less costly than others. By this method, stable transgene integration is achieved, mostly with single-copy integration [65]. Due to all these properties, to date, it is a widely accepted method of amongst all known methods. The only drawback of this method is, it requires a binary vector, and it incorporates an alien gene into the plant genome.

\section{Floral-dip or pollen-tube pathway method}

The earlier plasmids was either directly applied onto the surface of stigma or mixed with pollen and then applied to their receptive stigma [66]. Further, various parameters were optimized for efficient gene transfer such as wounding and dipping of flowers having male and female organs into Agrobacterium suspension. For the successful floral transformation of plants, the stage of plants is crucial. Apart from various physical parameters such as media composition, $\mathrm{pH}$, optical density, temperature, and humidity, molecular factors such as choice of the promoter, gene size, and vector types are also important. The well-known constitutive promoter such as CaMV35S and Arabidopsis UBI10 was also involved for increased editing efficiency. UBI10 promoter was found more efficient in the germline of Arabidopsis [5]. Further, the germline-specific promoter, such as MGE1, YAO, RPS5a, AG, and ICU2 promoters, was used for efficient editing [5]. The highest editing efficiency was obtained using RPS5a and YAO promoters [5]. Egg cellspecific promoter EC1.2 and EC1.2::EC1.1 has also exhibited substantial editing efficiency with CRISPR/Cas9 in Arabidopsis [67]. To date, the floral dip-mediated genome editing is limited to only Arabidopsis [5, 67]. However, the successful plant genetic transformation was done in flax, tomato, radish, Brassica rapa, and wheat, Setaria viridis using floral dip [68-71]. The 50$60 \%$ transformation efficiency was reported in flax, which is higher than those reported for Arabidopsis using the floral-dip method of gene transfer [69].

The main advantage of this delivery method did not require a plant tissue culture facility. Floral-dipmediated delivery of CRISPR/Cas9 components is cost- effective and straightforward. This method is most widely and commonly used for Arabidopsis genome editing across the world. The drawback of floral-dip-mediated delivery of CRISPR/Cas9 components is limited to few plants such as Arabidopsis, flax, and tomato etc, with less efficiency due to limited flower and seed formation.

\section{Knock-in through the sequential floral-dip method}

Site-directed insertion of the desired gene or promoter or desired segment of DNA at a specific location by CRISPR/ Cas9 is in demand. This has been successfully demonstrated in tomato, maize, wheat, and potato. In this, a donor template or donor vector is required, which consists of the left and right homology arm. For example, one of the donor vectors consists of two T-MLO homology arms and a GFP coding sequence. This constructed GFP donor vector transferred into wheat protoplast for GFP knock-in along with the CRISPR/Cas9 vector [38]. CRISPR/Cas9 component, along with the donor vector, was used in soybean callus. This donor DNA is consists of soybean specific promoter and HptII gene that confer hygromycin resistance [33]. The knock-in using CRISPR can be done into the germline cells or other regenerative cells with the help of the donor vector. The Arabidopsis line carrying Cas9 was used for sequential floral-dip method of transformation using germline-specific promoters such as DD45, Lat52, YAO, and CDC45 promoter [72]. The Cas9 regulated with DD45 promoter was found more efficient for knock-in, with the high rate of editing in egg cells or early embryos as compared to other regenerative tissues [72]. The floral-dip method of gene transfer has been demonstrated in various crops such as wheat [68], flax [69], radish [70], and tomato [71]. The Cas9, driven by egg cell or embryo-specific promoters along with the desired donor DNA template, might lead to efficient knock-in of targeted genes in different crops.

\section{Nanoparticle-mediated delivery}

To date, various efforts have been made to uptake different nanoparticles into dicot and monocot plant cells. The direct uptake of numerous nanoparticles includes mesoporous silica nanoparticles [73], carbon nanotubes [74], quantum dots [75], and metal/metal oxide NPs [76, 77]. The success of silicon carbide whisker-mediated genetic transformation of various crops such as maize [78], cotton [79], and rice [80] suggests that CRISPR/ Cas9-mediated genome editing will be useful to generate transgene-free plants. Cas9/gRNA ribonucleoproteins, along with appropriate nanoparticles, can be delivered into regenerative tissues. The multiple gRNAs, along with appropriate promoters and terminators, into a single plant transformation vector, are required for modulating multiple pathways (Fig. 3). However, the large size of a construct or multiple gRNAs will be tedious for 


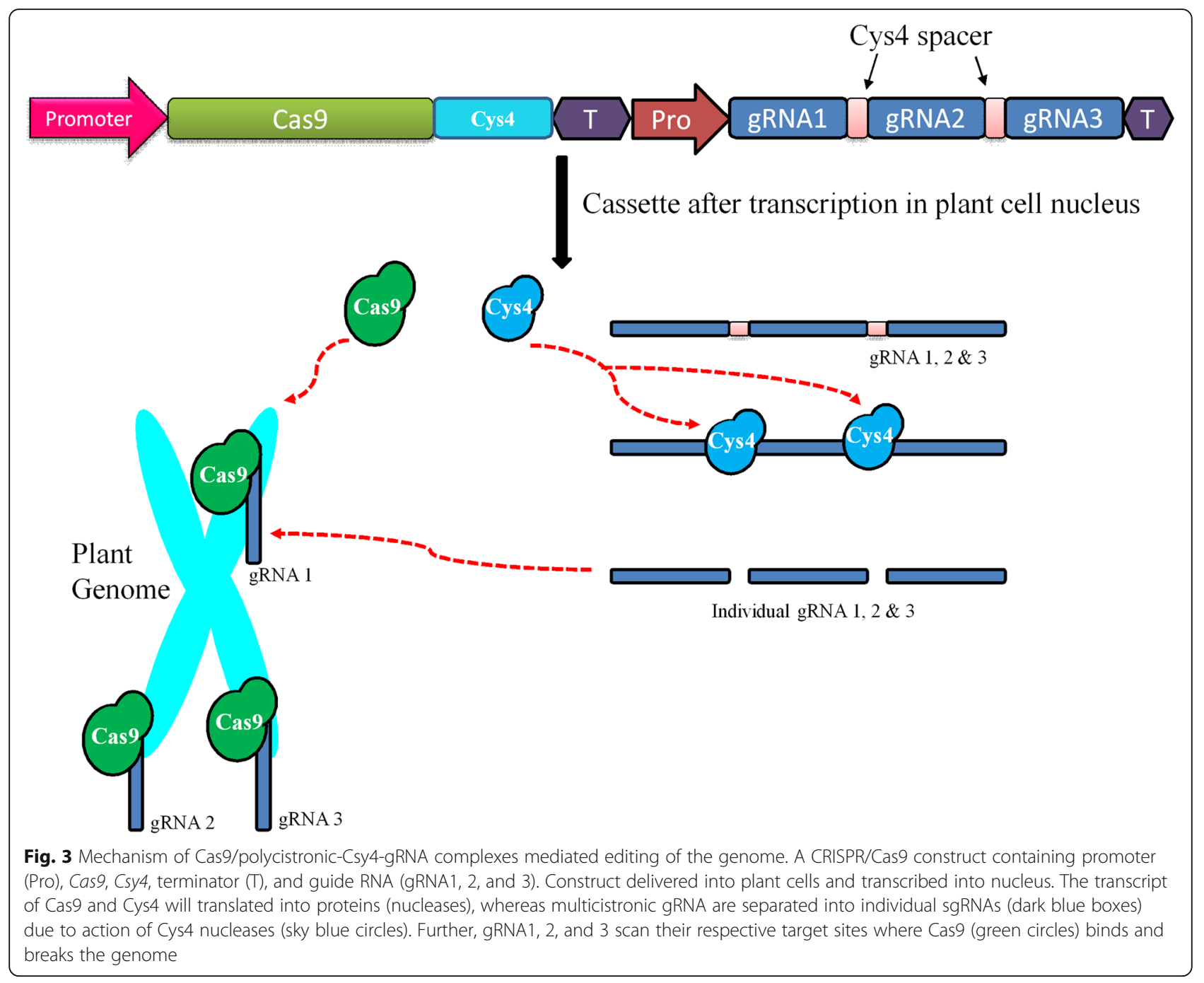

delivering into plant cells. Therefore, polycistronictRNA-gRNA or polycistronic-Csy4-gRNA, along with nanoparticles, will be effective for multiple editing via non-transgenic approaches. The success of every delivery method depends upon the method used as well as successive regeneration into whole plants. The plant protoplast is the primary target for the delivery of CRISPR/ Cas9 components. However, less regeneration frequency leads to lower editing efficiency.

The main advantage of nanoparticle-mediated delivery of CRISPR/Cas9 components is not limited to a protoplast. We can directly deliver it into plant regenerative tissues. The disadvantage of this method is less efficient and needs suitable nanoparticles with high carrying capacity CRISPR/Cas9 components.

\section{Pollen magnetofection-mediated delivery}

Magnetofection is a technique of genetic transformation that utilizes the magnetic force for the uptake of vector allied with magnetic nanoparticles (MNP). In this method, positively charged polyethyleneimine-coated $\mathrm{Fe}_{3} \mathrm{O}_{4}$ MNPs and negatively charged vector are used to form MNP-DNA complexes. Further, the pollens are mixed with the complexes, and a magnetic field is applied. Then, the pollens were applied for pollination. This technology has successfully applied to cotton [81]. Nowadays, two methods are frequently applied for delivery of CRISPR/Cas9 components: the first one is CRISPR/Cas9 vectors, and the second is vector/DNA less CRISPR/Cas9 system. The vector/DNA-free editing by magnetofection will be beneficial for generating nontransgenic crops. The following two methods are used to achieve this: (i) Transcribed sgRNA and Cas9 mRNA: The gRNA and Cas9 are transcribed in vitro and then coated with MNP and delivered to stigma or protoplast. For in vitro transcription, gRNA and Cas9 are regulated by a T7 promoter. The T7 RNA polymerase can be used for in vitro transcription and finally treated with DNase I. For example, in vitro mRNA transcripts of gRNA and Cas9 were co-bombarded into wheat calli [82]. (ii) 
Transcribed sgRNA and purified Cas9 protein: Cas9 protein and transcribed sgRNA can be attached to the MNP and then mix with pollens. Further, these can be transferred onto the stigma for fertilization or directly regenerated by tissue culture for mutant haploid production. Vector/DNA-free genome editing was shown in Arabidopsis, tobacco, lettuce, and rice [29]. Fruit crops such as grapevine and apple were also edited by the vector-free method using CRISPR/Cas9 ribonucleoproteins [83]. In maize, these complexes were bombarded in the embryo, and transgene-free mutant was recovered [32].

To date, there was no report for pollen magnetofectionmediated genome editing. However, the advantage of this method is we can directly transfer the CRISPR/Cas9 ribonucleoproteins into pollens. This can save the time required for tissue culture and selection of transgenic.

\section{Factors affecting editing efficiency}

Different factors such as selectable marker, promoter regulating Cas9 gene and gRNA, Agrobacterium strain, PEG concentration, temperature, and bombardment pressures might affect mutation frequency. Higher expression of the Cas 9 gene and gRNA depends on the correct promoter, and finally, it leads to higher mutation. Recent studies showed that U6 small nuclear promoter (U6) and human H1 Pol III promoter (H1) promoters efficiently express gRNA. H1, U6, and U3 promoters are RNA polymerase III promoters that efficiently transcribe short non-coding transcripts. Therefore, these promoters are used to regulate $75 \mathrm{bp}$ short gRNA. However, the $\mathrm{H} 1$ promoter is more useful than U6 because transcription can be initiated with any nucleotide in the case of $\mathrm{H} 1$ promoter, whereas U6 requires a $\mathrm{G}$ nucleotide. The robust $\mathrm{H} 1$ polymerase III promoter efficiently regulates gRNA [84]. T7, T3, and SP6 promoters are RNA polymerase promoters characterized from bacteriophage. These promoters require initiating $\mathrm{G}$ nucleotide and used in in vitro transcription of gRNA and Cas9 [84]. The strength of the promoter estimated by comparing the percentage of mutation which occurs in the plant. In Zea mays, the ubiquitin promoter showed more efficiency than the CaMV35S [85]. Ubiquitin (Ubi) and Cauliflower mosaic virus (CaMV35S) promoters are well known as a constitutive promoter, and it expresses every tissue of plants. DNA-dependent RNA polymerase III (pol III)/U3 promoter and pol III terminator are used to drive the gRNA expression. pRGE3 and pRGE6 vectors use Oryza sativum pol III (SnoRNA U6 and U3) promoter. The Arabidopsis AtU6 and AtU3 promoters are frequently used to regulate gRNA in dicots plants, whereas OsU6 and OsU3 are used in monocot plants $[12,28,86]$.
Cas9 from Streptococcus pyogenes (SpCas9)was more active at $37^{\circ} \mathrm{C}$ as compared to $22^{\circ} \mathrm{C}$ during the in vitro assay. SpCas9 was used for genome editing in Arabidopsis. It showed higher editing efficiency when Arabidopsis were subjected to heat stress at $37^{\circ} \mathrm{C}$ as compared to $22^{\circ} \mathrm{C}$ [87]. Genome editing using $\mathrm{Cpf1}$ requires higher temperatures for efficient editing efficiency. The Lachnospiraceae bacterium Cpf1 showed less editing efficiency at $22^{\circ} \mathrm{C}$, whereas it showed $100 \%$ editing efficiency at $28^{\circ} \mathrm{C}$ [88]. CRISPR/Cas9-mediated genome editing of citrus also showed higher editing efficiency when plants were subjected to heat stress at $37^{\circ} \mathrm{C}$ [87]. These all results suggest that temperature is also an essential factor affecting the mutation frequency.

\section{Advancement in CRISPR technology and future perspective}

Recent advancements in CRISPR technology are CRISPR interference (CRISPRi) and CRISPR mediated activation (CRISPRa). Different types of vectors for this are available commercially to open up new ways in genome engineering. CRISPRi and CRISPRa had been used in plants to control gene expression at the transcriptional level $[89,90]$. The deadCas9 (dCas9) catalytically inactive is used in these techniques. The dCas 9 alone or fused with a transcriptional repressor is used in CRISPRi technology. This chimeric dCas9 protein binds at their respective target site within the promoter region. Due to this, RNA polymerase activity is suppressed, and finally, the transcription is blocked [7, 89, 90]. In CRISPRa, dCas9 fused with transcriptional activators and therefore enhanced transcription as compared to normal promoter strength [28]. Cas9 nickases are produced by mutating RuvC (D10A) and HNH (H840A) domains of wild-type Cas9 [91]. It can cleave only one strand of DNA and therefore used as a paired to break both strands. Paired Cas9 nickases have less off-target activity as compared to wild-type Cas9 plants such as Arabidopsis and rice [91]. Cas9 nickases are useful for targeted gene insertion in plants.

All these chimeric proteins and variant Cas9 proteins need to be delivered into plant cells efficiently. To date, this has been achieved by Agrobacterium-mediated genetic transformation. Agrobacterium-mediated transformation of rice, tobacco, and Arabidopsis was done for delivery CRISPRi and CRISPRa construct [28, 61]. The delivery of this chimeric Cas9 protein along with multiple gRNA without using vector will be useful for crop improvement. However, the delivery of in vitro transcribed polycistronic tRNA-gRNA or Cys4-gRNA unit along with purified Cas9 would be a challenge. Probably, pollen magnetofection mediated and nanoparticlemediated delivery will solve these issues and generate non-transgenic mutant plants. 


\section{Conclusion}

The high efficiency of genome editing depends upon various factors. However, the most important is the type of delivery method used. In banana, 100\% editing efficiency was reported using Agrobacterium-mediated delivery of CRISPR/Cas9 components [62]. The success of every delivery method depends upon the tissue type and successive regeneration into whole plants. These concerns of regeneration depend upon the nature of plant species, tissue type, and culture method. Therefore, this publication emphasized the need to develop new methods for delivery of CRISPR/Cas9 components such as nanoparticle-mediated delivery and pollen magnetofection mediated delivery. These two potential methods of delivery into pollen or directly into the meristematic region would allow researchers to omit the time consuming and laborious tissue culture. The authors expected these novel delivery methods would boost up the CRISPR/Cas technologies in agriculture. The crops with altered genome will be cross the barrier of ethical as well as regulatory issues because it does not require any vector of DNA for editing.

\section{Abbreviations}

gRNA: Guide RNA; RGNs: Site-specific nucleases; PAM: Protospacer Adjacent Motif; DSBs: Double-stranded breaks; HDR: Homologous direct repair; NHEJ: Non-homologous end joining; PEG: Polyethylene glycol; MNP: Magnetic nanoparticles

\section{Acknowledgements}

DS is thankful to the Department of Science and Technology, Government of India for providing Inspire Fellowship (IF160264). PJ and VRA are thankful to the University Grants Commission-Special Assistance Programme, Government of India for providing financial support (F-5-24/2015/DRS-II). SA is thankful for the Council of Scientific \& Industrial Research, Government of India for providing CSIR-Emeritus Scientist (21(1067)/19/EMR-II). We are thankful to Dr. Sudhir P Singh, CIAB Mohali for critical suggestion and manuscript improvement.

\section{Authors' contributions}

SD, PJ, and AA wrote the manuscript. AA conceived the idea. AA, VRA, and SA edited the manuscript. All authors have read and approved the manuscript.

\section{Funding}

No funding was used for this article.

\section{Availability of data and materials}

Not applicable for this article.

Ethics approval and consent to participate

Not applicable for this article.

\section{Consent for publication}

Not applicable for this article.

\section{Competing interests}

Authors declare that they have no conflict of interest.
Received: 19 December 2019 Accepted: 4 June 2020

Published online: 07 July 2020

\section{References}

1. Molinar, R. (2012). Traditional plant breeding vs. genetic engineering-a primer. https://www.farmprogress.com/

2. Mahfouz MM, Piatek A, Stewart CN Jr (2014) Genome engineering via TALENs and CRISPR/Cas9 systems: challenges and perspectives. Plant Biotechnol J 12(8):1006-1014

3. Ain QU, Chung JY, Kim YH (2015) Current and future delivery systems for engineered nucleases: ZFN, TALEN and RGEN. J Control Release 205:120_ 127

4. Andersson M, Turesson $H_{1}$ Olsson N, Fält AS, Ohlsson P, Gonzalez MN, Hofvander P (2018) Genome editing in potato via CRISPR-Cas9 ribonucleoprotein delivery. Physiol Plant 164(4):378-384

5. Castel B, Tomlinson L, Locci F, Yang Y, Jones JD (2019) Optimization of TDNA architecture for Cas9-mediated mutagenesis in Arabidopsis. PLoS One 14(1):e0204778

6. Hsu PD, Lander ES, Zhang F (2014) Development and applications of CRISPR-Cas9 for genome engineering. Cell 157(6):1262-1278

7. Bortesi L, Fischer R (2015) The CRISPR/Cas9 system for plant genome editing and beyond. Biotechnol Adv 33(1):41-52

8. Belhaj KA, Chaparro-Garcia S, Kamoun NV (2013) Plant genome editing made easy: targeted mutagenesis in model and crop plants using the CRISPR/Cas system. Plant Methods 9(1):1

9. Belhaj KA, Chaparro-Garcia S, Kamoun NJ, Patron V, Nekrasov (2015) Editing plant genomes with CRISPR/Cas9. Curr Opin Biotechnol 32:76-84

10. Cong L, Ran FA, Cox D, Lin S, Barretto R, Habib N, Hsu PD, Wu X, Jiang W, Marraffini L, Zhang F (2013) Multiplex genome engineering using CRISPR/ Cas systems. Science:1231143

11. Cermak T, Curtin SJ, Gil-Humanes J, Čegan R, Kono TJ, Konečná E, Voytas DF (2017) A multi-purpose toolkit to enable advanced genome engineering in plants. Plant Cell tpc-00922

12. Xie K, Minkenberg B, Yang Y (2015) Boosting CRISPR/Cas9 multiplex editing capability with the endogenous tRNA-processing system. Proc Natl Acad Sci 112(11):3570-3575

13. Kaur N, Alok A, Kaur N, Pandey P, Awasthi P, Tiwari S (2018) CRISPR/Cas9mediated efficient editing in phytoene desaturase (PDS) demonstrates precise manipulation in banana cv. Rasthali genome. Func Integ Genom 18(1):89-99

14. Upadhyay SK, Kumar J, Alok A, Tuli R (2013) RNA-guided genome editing for target gene mutations in wheat. G3: Genes, Genomes. Genetics 3(12): 2233-2238

15. Malnoy M, Viola R, Jung MH, Koo OJ, Kim S, Kim JS, Velasco R, Nagamangala Kanchiswamy C (2016) DNA-free genetically edited grapevine and apple protoplast using CRISPR/Cas9 ribonucleoproteins. Front Plant Sci 7:1904

16. Murovec J, Guček K, Bohanec B, Avbelj M, Jerala R (2018) DNA-free genome editing of Brassica oleracea and B. rapa protoplasts using CRISPR-Cas9 ribonucleoprotein complexes. Front Plant Sci 9:1594

17. Tian S, Jiang L, Gao Q, Zhang J, Zong M, Zhang H, Ren Y, Guo S, Gong G, Liu F (2016) Efficient CRISPR/Cas9-based gene knockout in watermelon. Plant Cell Rep 36(3):399-406

18. Sun X, Hu Z, Chen R, Jiang Q, Song G, Zhang H, Xi Y (2015) Targeted mutagenesis in soybean using the CRISPR-Cas9 system. Sci Rep 5:10342

19. Xie K, Yang Y (2013) RNA-guided genome editing in plants using a CRISPRCas system. Mol Plant 6(6):1975-1983

20. Jiang W, Zhou H, Bi H, Fromm M, Yang B, Weeks DP (2013) Demonstration of CRISPR/Cas9/sgRNA-mediated targeted gene modification in Arabidopsis, tobacco, sorghum and rice. Nucleic Acids Res 41(20):e188

21. Shan Q, Wang Y, Li J, Zhang Y, Chen K, Liang Z, Zhang K, Liu J, Xi JJ, Qiu J-L (2013) Targeted genome modification of crop plants using a CRISPR-Cas system. Nat Biotechnol 31(8):686-688

22. Xu J, Kang BC, Naing AH, Bae SJ, Kim JS, Kim H, Kim CK (2020) CRISPR/Cas9mediated editing of 1-aminocyclopropane-1-carboxylate oxidase1 enhances Petunia flower longevity. Plant Biotechnol J 18(1):287-297

23. Collonnier C, Epert A, Mara K, Maclot F, Guyon-Debast A, Charlot F, White C, Schaefer DG, Nogué F (2016) CRISPR-Cas9-mediated efficient directed mutagenesis and RAD51-dependent and RAD51-independent gene targeting in the moss Physcomitrella patens. Plant Biotechnol J 15(1):122131 
24. Arndell $T$, Sharma $N$, Langridge $P$, Baumann $U$, Watson-Haigh NS, Whitford $R$ (2019) gRNA validation for wheat genome editing with the CRISPR-Cas9 system. BMC Biotechnol 19(1):1-12

25. Liang Z, Zhang K, Chen K, Gao C (2014) Targeted mutagenesis in Zea mays using TALENs and the CRISPR/Cas system. J Genet Genom 41(2):63-68

26. Li Y, Zhu J, Wu H, Liu C, Huang C, Lan J, Zhao Y, Xie C (2019) Precise base editing of non-allelic acetolactate synthase genes confers sulfonylurea herbicide resistance in maize. Crop J

27. Lowder L, Malzahn A, Qi Y (2016) Rapid evolution of manifold CRISPR systems for plant genome editing. Front Plant Sci 7:1683

28. Lowder LG, Zhang D, Baltes NJ, Paul JW, Tang X, Zheng X, Voytas DF, Hsieh T-F, Zhang Y, Qi Y (2015) A CRISPR/Cas9 toolbox for multiplexed plant genome editing and transcriptional regulation. Plant Physiol:00636

29. Woo JW, Kim J, Kwon SI, Corvalán C, Cho SW, Kim H, Kim SG, Kim ST, Choe S, Kim JS (2015) DNA-free genome editing in plants with preassembled CRISPR-Cas9 ribonucleoproteins. Nat Biotechnol 33(11):1162

30. Kim H, Kim ST, Ryu J, Kang BC, Kim JS, Kim SG (2017) CRISPR/Cpf1-mediated DNA-free plant genome editing. Nat Commun 8:14406

31. Waltz E (2016) Gene-edited CRISPR mushroom escapes US regulation. Nature 532(7599):293-293 https://doi.org/10.1038/nature.2016.19754

32. Svitashev S, Young JK, Schwartz C, Gao H, Falco SC, Cigan AM (2015) Targeted mutagenesis, precise gene editing, and site-specific gene insertion in maize using Cas9 and guide RNA. Plant Physiol 169(2):931-945

33. Li Z, Liu Z-B, Xing A, Moon BP, Koellhoffer JP, Huang L, Ward RT, Clifton E, Falco SC, Cigan AM (2015) Cas9-guide RNA directed genome editing in soybean. Plant Physiol:00783

34. Kapusi E, Corcuera-Gómez M, Melnik S, Stoger E (2017) Heritable genomic fragment deletions and small indels in the putative ENGase gene induced by CRISPR/Cas9 in barley. Front Plant Sci 8:540

35. Dong OX, Yu S, Jain R, Zhang N, Duong PQ, Butler C, Li Y, Lipzen A, Martin JA, Barry KW, Schmutz J (2020) Marker-free carotenoid-enriched rice generated through targeted gene insertion using CRISPR-Cas9. Nat Commun 11(1):1-10

36. Banakar R, Eggenberger AL, Lee K, Wright DA, Murugan K, Zarecor S, Lawrence-Dill CJ, Sashital DG, Wang K (2019) High-frequency random DnA insertions upon co-delivery of CRISPR-Cas9 ribonucleoprotein and selectable marker plasmid in rice. Sci Rep 9(1):1-13

37. Shan Q, Wang Y, Li J, Gao C (2014) Genome editing in rice and wheat using the CRISPR/Cas system. Nat Protoc 9(10):2395

38. Wang Y, Cheng X, Shan Q, Zhang Y, Liu J, Gao C, Qiu J-L (2014) Simultaneous editing of three homoeoalleles in hexaploid bread wheat confers heritable resistance to powdery mildew. Nat Biotechnol 32(9):947951

39. Li C, Zong Y, Wang Y, Jin S, Zhang D, Song Q, Gao C (2018) Expanded base editing in rice and wheat using a Cas9-adenosine deaminase fusion. Genome Biol 19(1):59

40. Baltes NJ, Gil-Humanes J, Voytas DF (2017) Chapter one - genome engineering and agriculture: opportunities and challenges. In: Weeks DP, Yang B (eds) Progress in Molecular Biology and Translational Science, vol 149. Academic Press, pp 1-26

41. Li J-F, Norville JE, Aach J, McCormack M, Zhang D, Bush J, Church GM, Sheen J (2013) Multiplex and homologous recombination-mediated genome editing in Arabidopsis and Nicotiana benthamiana using guide RNA and Cas9. Nat Biotechnol 31(8):688-691

42. Feng Z, Zhang B, Ding W, Liu X, Yang D-L, Wei P, Cao F, Zhu S, Zhang F, Mao Y (2013) Efficient genome editing in plants using a CRISPR/Cas system. Cell Res 23(10):1229

43. Kaur N, Alok A, Kumar P, Kaur N, Awasthi P, Chaturvedi S, Pandey P, Pandey A, Pandey AK, Tiwari S (2020) CRISPR/Cas9 directed editing of lycopene epsilon-cyclase modulates metabolic flux for $\beta$-carotene biosynthesis in banana fruit. Metab Eng 59:76-86

44. Wang L, Chen S, Peng A, Xie Z, He Y, Zou X (2019) CRISPR/Cas9-mediated editing of CsWRKY22 reduces susceptibility to Xanthomonas citri subsp. citri in Wanjincheng orange (Citrus sinensis (L.) Osbeck). Plant Biotechnol Rep 13(5):501-510

45. Chandrasekaran J, Brumin M, Wolf D, Leibman D, Klap C, Pearlsman M Sherman A, Arazi T, Gal-On A (2016) Development of broad virus resistance in non-transgenic cucumber using CRISPR/Cas9 technology. Mol Plant Pathol 17(7):1140-1153

46. Jacobs TB, LaFayette PR, Schmitz RJ, Parrott WA (2015) Targeted genome modifications in soybean with CRISPR/Cas9. BMC Biotechnol 15(1):16
47. Wang Z, Wang S, Li D, Zhang Q, Li L, Zhong C, Huang H (2018) Optimized paired-sgRNA/Cas9 cloning and expression cassette triggers high-efficiency multiplex genome editing in kiwifruit. Plant Biotechnol J

48. Wang L, Wang L, Tan Q, Fan Q, Zhu H, Hong Z, Zhang Z, Duanmu D (2016) Efficient inactivation of symbiotic nitrogen fixation related genes in Lotus japonicus using CRISPR-Cas9. Front Plant Sci 7:1333

49. Sugano SS, Nishihama R, Shirakawa M, Takagi J, Matsuda Y, Ishida S, Kohchi T (2018) Efficient CRISPR/Cas9-based genome editing and its application to conditional genetic analysis in Marchantia polymorpha. PLoS One 13:10

50. Michno JM, Wang X, Liu J, Curtin SJ, Kono TJ, Stupar RM (2015) CRISPR/Cas mutagenesis of soybean and Medicago truncatula using a new web-tool and a modified Cas9 enzyme. GM Crops Food 6(4):243-252

51. Meng Y, Hou Y, Wang H, Ji R, Liu B, Wen J, Niu L, Lin H (2017) Targeted mutagenesis by CRISPR/Cas9 system in the model legume Medicago truncatula. Plant Cell Rep 36(2):371-374

52. Yin K, Han T, Liu G, Chen T, Wang Y, Yu AYL, Liu Y (2015) A geminivirusbased guide RNA delivery system for CRISPR/Cas9 mediated plant genome editing. Sci Rep 5:14926

53. Gao J, Wang G, Ma S, Xie X, Wu X, Zhang X, Wu Y, Zhao P, Xia Q (2015) CRISPR/Cas9-mediated targeted mutagenesis in Nicotiana tabacum. Plant Mol Biol 87(1-2):99-110

54. Shufen C, Yicong C, Baobing F, Guiai J, Zhonghua S, Ju LUO, Shaoqing T, Jianlong W, Peisong $\mathrm{H}$, Xiangjin W (2019) Editing of rice isoamylase gene ISA1 provides insights into its function in starch formation. Rice Sci 26(2):7787

55. Fan D, Liu T, Li C, Jiao B, Li S, Hou Y, Luo K (2015) Efficient CRISPR/Cas9mediated targeted mutagenesis in Populus in the first generation. Sci Rep 5:12217

56. Zhou Z, Tan H, Li Q, Chen J, Gao S, Wang Y, Zhang L (2018) CRISPR/Cas9mediated efficient targeted mutagenesis of RAS in Salvia miltiorrhiza. Phytochemistry 148:63-70

57. Li X, Wang Y, Chen S, Tian H, Fu D, Zhu B, Luo Y, Zhu H (2018) Lycopene is enriched in tomato fruit by CRISPR/Cas9-mediated multiplex genome editing. Front Plant Sci 9:559

58. Bari VK, Nassar JA, Kheredin SM, Gal-On A, Ron M, Britt A, Steele D, Yoder J, Aly R (2019) CRISPR/Cas9-mediated mutagenesis of CAROTENOID CLEAVAGE DIOXYGENASE 8 in tomato provides resistance against the parasitic weed Phelipanche aegyptiaca. Sci Rep 9(1):1-12

59. Butler NM, Atkins PA, Voytas DF, Douches DS (2015) Generation and inheritance of targeted mutations in potato (Solanum tuberosum L.) using the CRISPR/Cas system. PLoS One 10(12):e0144591

60. Char SN, Neelakandan AK, Nahampun H, Frame B, Main M, Spalding MH, Becraft PW, Meyers BC, Walbot V, Wang K (2016) An Agrobacteriumdelivered CRISPR/Cas9 system for high-frequency targeted mutagenesis in maize. Plant Biotechnol J 15(2):257-268

61. Xing H-L, Dong L, Wang Z-P, Zhang H-Y, Han C-Y, Liu B, Wang X-C, Chen QJ (2014) A CRISPR/Cas9 toolkit for multiplex genome editing in plants. BMC Plant Biol 14(1):1

62. Naim F, Dugdale B, Kleidon J, Brinin A, Shand K, Waterhouse P, Dale J (2018) Gene editing the phytoene desaturase alleles of Cavendish banana using CRISPR/Cas9. Transgenic Res 27(5):451-460

63. Jia H, Wang N (2014) Targeted genome editing of sweet orange using Cas9/sgRNA. PLoS One 9(4):e93806

64. Zhou X, Jacobs TB, Xue L, Harding SA, Tsai CJ (2015) Exploiting SNPs for biallelic CRISPR mutations in the outcrossing woody perennial Populus reveals 4-coumarate: CoA ligase specificity and redundancy. New Phytol 208(2):298-301

65. Travella S, Ross SM, Harden J, Everett C, Snape JW, Harwood WA (2005) A comparison of transgenic barley lines produced by particle bombardment and Agrobacterium-mediated techniques. Plant Cell Rep 23(12):780-789

66. Ohta $Y$ (1986) High-efficiency genetic transformation of maize by a mixture of pollen and exogenous DNA. Proc Natl Acad Sci 83(3):715-719

67. Wang S, Zhang S, Wang W, Xiong X, Meng F, Cui X (2015) Efficient targeted mutagenesis in potato by the CRISPR/Cas9 system. Plant Cell Rep 34(9): 1473-1476

68. Zale JM, Agarwal S, Loar S, Steber CM (2009) Evidence for stable transformation of wheat by floral dip in Agrobacterium tumefaciens. Plant Cell Rep 28(6):903-913

69. Bastaki NK, Cullis CA (2014) Floral-dip transformation of flax (Linum usitatissimum) to generate transgenic progenies with a high transformation rate. JoVE 94:e52189 
70. Curtis IS, Nam HG (2001) Transgenic radish (Raphanus sativus L. longipinnatus Bailey) by floral-dip method-plant development and surfactant are important in optimizing transformation efficiency. Transgenic Res 10(4):363-371

71. Sharada MS, Kumari A, Pandey AK, Sharma S, Sharma P, Sreelakshmi Y, Sharma R (2017) Generation of genetically stable transformants by Agrobacterium using tomato floral buds. Plant Cell Tiss Org Cult 129(2):299312

72. Miki D, Zhang W, Zeng W, Feng Z, Zhu JK (2018) CRISPR/Cas9-mediated gene targeting in Arabidopsis using sequential transformation. Nat Commun 9(1):1967

73. Hussain HI, Yi Z, Rookes JE, Kong LX, Cahill DM (2013) Mesoporous silica nanoparticles as a biomolecule delivery vehicle in plants. J Nanopart Res 15(6):1676

74. Liu Q, Chen B, Wang Q, Shi X, Xiao Z, Lin J, Fang X (2009) Carbon nanotubes as molecular transporters for walled plant cells. Nano Lett 9(3): 1007-1010

75. Koo Y, Wang J, Zhang Q, Zhu H, Chehab EW, Colvin VL, Braam J (2014) Fluorescence reports intact quantum dot uptake into roots and translocation to leaves of Arabidopsis thaliana and subsequent ingestion by insect herbivores. Environ Sci Technol 49(1):626-632

76. Kurepa J, Paunesku T, Vogt S, Arora H, Rabatic BM, Lu J, Smalle JA (2010) Uptake and distribution of ultrasmall anatase $\mathrm{TiO} 2$ Alizarin red $\mathrm{S}$ nanoconjugates in Arabidopsis thaliana. Nano Lett 10(7):2296-2302

77. Singh J, Kumar S, Alok A, Upadhyay SK, Rawat M, Tsang DC, Kim KH (2019) The potential of green synthesized zinc oxide nanoparticles as nutrient source for plant growth. J Clean Prod 214:1061-1070

78. Frame BR, Drayton PR, Bagnall SV, Lewnau CJ, Bullock WP, Wilson HM et al (1994) Production of fertile transgenic maize plants by silicon carbide whisker-mediated transformation. Plant J 6(6):941-948

79. Arshad M, Zafar Y, Asad S (2013) Silicon carbide whisker-mediated transformation of cotton (Gossypium hirsutum L.). Transgenic Cotton:79-92

80. Matsushita J, Otani M, Wakita Y, Tanaka O, Shimada T (1999) Transgenic plant regeneration through silicon carbide whisker-mediated transformation of rice (Oryza sativa L.). Breed Sci 49(1):21-26

81. Zhang R, Meng Z, Abid MA, Zhao X (2019) Novel pollen magnetofection system for transformation of cotton plant with magnetic nanoparticles as gene carriers. In: Transgenic Cotton. Humana Press, New York, pp 47-54

82. Zhang Y, Liang Z, Zong Y, Wang Y, Liu J, Chen K, Gao C (2016) Efficient and transgene-free genome editing in wheat through transient expression of CRISPR/Cas9 DNA or RNA. Nat Commun 7:12617

83. Malnoy M, Viola R, Jung MH, Koo OJ, Kim S, Kim JS, Nagamangala Kanchiswamy C (2016) DNA-free genetically edited grapevine and apple protoplast using CRISPR/Cas9 ribonucleoproteins. Front Plant Sci 7:1904

84. Ranganathan V, Wahlin K, Maruotti J, Zack DJ (2014) Expansion of the CRISPR-Cas9 genome targeting space through the use of $\mathrm{H} 1$ promoterexpressed guide RNAs. Nat Commun 5:4516

85. Azhakanandam K, Zhang ZJ (2015) Sorghum transformation: achievements, challenges, and perspectives. In: Recent Advancements in Gene Expression and Enabling Technologies in Crop Plants. Springer, New York, pp 291-312

86. Nowak CM, Lawson S, Zerez M, Bleris L (2016) Guide RNA engineering for versatile Cas9 functionality. Nucleic Acids Res 44(20):9555-9564

87. LeBlanc C, Zhang F, Mendez J, Lozano Y, Chatpar K, Irish VF, Jacob Y (2018) Increased efficiency of targeted mutagenesis by CRISPR/Cas9 in plants using heat stress. Plant J 93(2):377-386

88. Alok A, Sandhya D, Jogam P, Rodrigues V, Bhati KK, Sharma H, Kumar J (2020) The rise of the CRISPR/Cpf1 system for efficient genome editing in plants. Front Plant Sci 11:264

89. Qi LS, Larson MH, Gilbert LA, Doudna JA, Weissman JS, Arkin AP, Lim WA (2013) Repurposing CRISPR as an RNA-guided platform for sequencespecific control of gene expression. Cell 152(5):1173-1183

90. Gilbert LA, Horlbeck MA, Adamson B, Villalta JE, Chen Y, Whitehead EH, Q LS (2014) Genome-scale CRISPR-mediated control of gene repression and activation. Cell 159(3):647-661

91. Čermák T, Baltes NJ, Čegan R, Zhang Y, Voytas DF (2015) Highfrequency,precise modification of the tomato genome. Genome Biol 16(1): 232

\section{Publisher's Note}

Springer Nature remains neutral with regard to jurisdictional claims in published maps and institutional affiliations.

\section{Submit your manuscript to a SpringerOpen ${ }^{\odot}$ journal and benefit from:}

- Convenient online submission

- Rigorous peer review

- Open access: articles freely available online

- High visibility within the field

- Retaining the copyright to your article

Submit your next manuscript at $\boldsymbol{\nabla}$ springeropen.com 